\title{
Toxicity associated with combination oxaliplatin plus fluoropyrimidine with or without cetuximab in the MRC COIN trial experience
}

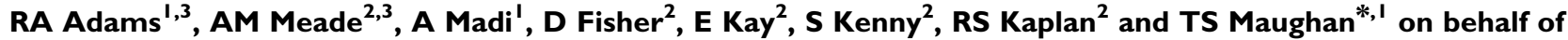 the COIN trial TMG and investigators}

'Velindre Hospital, Cardiff University, Velindre Road, Whitchurch, Cardiff CFI 4 2TL, UK; ${ }^{2}$ Medical Research Council Clinical Trials Unit, Euston Road, London, UK

\begin{abstract}
We present the preliminary toxicity data from the MRC COIN trial, a phase III randomised controlled trial of first-line therapy in advanced colorectal cancer, with particular reference to the addition of cetuximab to an oxaliplatin-fluoropyrimidine combination. A total of 804 patients were randomised between March 2005 and July 2006 from 78 centres throughout the United Kingdom. Patients were allocated to oxaliplatin plus fluoropyrimidine chemotherapy with or without the addition of weekly cetuximab. The choice of fluoropyrimidine (either 5-fluorouracil (5FU) or capecitabine) was decided by the treating physician and patient before randomisation. Toxicity data were collected from all patients. Two hundred and three patients received 5FU plus oxaliplatin (OxMdG, 25\%), 333 oxaliplatin + capecitabine (Xelox, 41\%), 102 received OxMdG + cetuximab (OxMdG + C, I3\%) and I66 Xelox + cetuximab (21\%). Percent grade 3/4 toxicities included diarrhoea 6, 15, 13 and 25\%, nausea/vomiting 3, 7, 7 and I4\% for OxMdG, Xelox, OxMdG $+\mathrm{C}$ and Xelox $+\mathrm{C}$, respectively. Sixty-day all-cause mortality was 6, 5, 5 and 7\%. Statistically significant differences were evident for patients receiving Xelox + cetuximab vs Xelox alone: diarrhoea relative risk (RR) I.69 (I.I7, 2.43 , $P=0.005)$ and nausea/vomiting RR 2.01 (I.16, 3.47, $P=0.012)$. The excess toxicity observed in the oxaliplatin-, capecitabine-, cetuximab-treated patients led the trial management group to conclude that a capecitabine dose adjustment was required to maintain safety levels when using this regimen.
\end{abstract}

British Journal of Cancer (2009) I 00, 25 I -258. doi: I0.1038/sj.bjc.6604877 www.bjcancer.com

(c) 2009 Cancer Research UK

Keywords: colorectal cancer; cetuximab; oxaliplatin; fluoropyrimidine; metastatic; first line

Advanced colorectal cancer (ACRC) causes over half a million deaths worldwide each year (http://www-dep.iarc.fr/). The treatment of ACRC is improving. Average survival has improved from 6 months with best supportive care alone, through 10-12 months with single-agent 5-fluorouracil (5FU) regimens up to 16-21 months in randomised trials using irinotecan, oxaliplatin and bevacizumab as well as $5 \mathrm{FU}$.

Cetuximab was licensed for use in epidermal growth factor receptor (EGFR)-expressing ACRC on the basis of the phase II BOND trial (Cunningham et al, 2004) in which 218 irinotecanrefractory patients received cetuximab and irinotecan combination therapy and 111 received cetuximab monotherapy. Although there was no chemotherapy are comparator arm in this trial, the data suggested no greater toxicity with the combination therapy than is expected in patients receiving single-agent irinotecan, other than a

\footnotetext{
*Correspondence: Professor TS Maughan, Velindre Hospital, Velindre Road, Whitchurch, Cardiff CFI4 2TL, UK;

E mail: tim.maughan@velindre-tr.wales.nhs.uk

Original data presented at ASCO (Chicago) 2007

${ }^{3}$ These authors contributed equally to this work

Received 8 August 2008; revised 4 December 2008; accepted I4 December 2008
}

higher proportion of patients with skin rash, a known side effect of EGFR inhibitor therapy. In the past year, the first randomised data on the combination of oxaliplatin and capecitabine in the first-line ACRC setting have been presented (Cassidy et al, 2008). In addition, first-line data are also now available from randomised trials investigating the addition of cetuximab to combination chemotherapy regimens containing both irinotecan (Van Cutsem et al, 2007) and oxaliplatin (Bokemeyer et al, 2007).

Antibodies targeting the EGFR have now shown efficacy in third-, second- and first-line therapies. In the Crystal trial, the addition of cetuximab to first-line irinotecan, infusional 5FU and folinic acid resulted in an $8 \%$ increase in response rate and a significant improvement in progression-free survival $(P=0.036)$ (Van Cutsem et al, 2007). The EPIC trial explored the use of irinotecan in combination with cetuximab in the second line, after irinotecan failure. This trial has recently been published and demonstrated a response rate with cetuximab and irinotecan of $16.4 \%(95 \%$ confidence interval (CI), 13.6-19.4), compared with 4.2\% (95\% CI, 2.8-6.0) with irinotecan alone $(P=0.0001)$ (Sobrero et al, 2008).

COIN is an open-label multicentre randomised controlled trial sponsored by the UK Medical Research Council (MRC) and coordinated by the MRC Clinical Trials Unit (MRC CTU), comparing two experimental arms with the control arm of oxaliplatin and fluoropyrimidine chemotherapy in the first-line 
treatment of ACRC. The fluoropyrimidine can be either capecitabine or bolus and infusional 5FU (in a modified FOLFOX regimen), and is chosen on a per-patient or per-institution basis before randomisation. Two primary questions are being addressed in the trial. The first question asks whether the addition of cetuximab to combination chemotherapy will increase overall survival, whereas the second asks whether intermittent chemotherapy treatment is comparable with continuous treatment to progression or cumulative toxicity.

The use of intermittent chemotherapy is controversial. A previous MRC trial showed no detriment in overall survival, with an improvement in toxicity and quality of life for patients receiving intermittent single-agent 5FU chemotherapy (Maughan et al, 2003). The Optimox 1 trial showed that discontinuation of oxaliplatin after six cycles of OxMdG, with continuation of infusional 5FU and subsequent reintroduction of oxaliplatin, resulted in equivalent disease control and survival (Tournigand et al, 2006). The Optimox 2 study, however, has raised doubts about the overall survival equivalence for patients given a chemotherapy-free interval. The original design of Optimox 2 was a phase III trial of 600 patients; however, accrual was discontinued when bevacizumab became available. At this stage, only 200 patients had been entered. The trial reported a non-significant overall median survival difference of 25 months with continuous infusional 5FU, compared with 19 months in the arm with chemotherapy-free intervals $(P=0.056)$ (Maindrault-Goebel et al, 2007). Owing to the failure of this trial to complete accrual as planned, and therefore answer the question originally posed, it is of utmost importance to obtain reliable phase III evidence of the relative benefits of continuous chemotherapy $v s$ intermittent chemotherapy with a chemotherapy-free interval in the treatment of ACRC. The COIN trial will be the largest randomised controlled trial of continuous $v s$ intermittent chemotherapy carried out to date. If successful, the intermittent chemotherapy strategy would not only benefit patients, who value time off chemotherapy very highly, but also open a new therapeutic window for the evaluation and use of novel agents to maintain disease control during intervals off chemotherapy.

In this paper, we present toxicity reported during the first 12 weeks of treatment for the first 804 patients randomised into the COIN trial. During the first 12 weeks, there is no difference in the treatment received by those patients randomised to the control arm of COIN and those randomised to intermittent therapy. Therefore, the focus of this paper is the toxicity experience in the first 12 weeks associated with the addition of cetuximab to two different oxaliplatin and fluoropyrimidine combination chemotherapy regimens. The 12 -week time point has been chosen as this is when patients randomised to intermittent therapy stop treatment. We propose to publish the longer term toxicity data (relating to the intermittent $v s$ continuous strategies) at a later date, together with quality of life and primary outcome data. In the meantime, however, we feel that the initial toxicity experience contains information of value to clinicians employing combinations of these widely available agents.

\section{PATIENTS AND METHODS}

\section{Accrual update}

The COIN trial opened to accrual in March 2005 and closed in May 2008 after completing accrual ahead of schedule; 2445 patients have been randomised from 111 centres in the United Kingdom and Republic of Ireland.

\section{Eligibility criteria}

Patients have histologically confirmed colorectal adenocarcinoma, with inoperable metastatic or locoregional disease. Eligibility criteria include disease measurable by RECIST (Therasse et al,
2000) WHO performance status $0-2$; no previous chemotherapy for metastatic disease; neutrophils $>1.5 \times 10^{9}$ per 1 , platelet count $>100 \times 10^{9}$ per 1 , serum bilirubin $\leq 1.25 \times$ upper limit of normal (ULN), alkaline phosphatase $\leqslant 5 \times$ ULN, calculated GFR or EDTA clearance $\geqslant 50 \mathrm{ml} \mathrm{min}^{-1}$; and age $>18$ years. Exclusion criteria include patients receiving combination chemotherapy before the resection of operable liver metastases (patients of uncertain operability are eligible), brain metastases, prior adjuvant chemotherapy with oxaliplatin and uncontrolled medical comorbidity likely to compromise treatment.

\section{Randomisation and treatment}

Patients were randomised centrally by the MRC CTU to one of the three trial arms on a 1:1:1 basis using a stratified, minimisation procedure. Treatment is started as soon as possible after randomisation and within 4 weeks of the baseline radiological disease assessment using CT scan. The trial arms are as follows:

\section{Arm A:}

Continuous chemotherapy (control arm) The chemotherapy regimen is a combination of oxaliplatin and a fluoropyrimidine.

The regimens are:

OxMdG: a combination of $l$-folinic acid ( $175 \mathrm{mg}$ i.v. over $2 \mathrm{~h}$ ) OR $d$,l-folinic acid ( $350 \mathrm{mg}$ i.v. over $2 \mathrm{~h}$ ), concurrent administration of oxaliplatin $\left(85 \mathrm{mg} \mathrm{m}^{-2}\right.$ i.v. over $\left.2 \mathrm{~h}\right)$ plus bolus $5 \mathrm{FU}$ $\left(400 \mathrm{mg} \mathrm{m}^{-2}\right)$ followed by a 46-h i.v. infusion of 5FU $2400 \mathrm{mg} \mathrm{m}^{-2}$ repeated every 2 weeks as used in the FOCUS trial (Seymour et al, 2007) or

Xelox: a combination of oxaliplatin $130 \mathrm{mg} \mathrm{m}^{-2}$ i.v. over $2 \mathrm{~h}$ on day 1 plus capecitabine $1000 \mathrm{mg} \mathrm{m}^{-2}$ b.d., p.o. on days $1-14$ repeated thrice weekly.

Patients continue chemotherapy until RECIST-defined progressive disease is identified, or the development of cumulative toxicity or because of patient choice to stop chemotherapy. Patients should have no more than a 3-week interval off treatment for any reason.

\section{Arm B:}

Continuous chemotherapy plus cetuximab Cetuximab is administered once weekly in combination with the chemotherapy schedules as above. On day 1 of the first cycle of chemotherapy, an initial loading dose of $400 \mathrm{mg} \mathrm{m}^{-2}$ cetuximab is given by 2-h i.v. infusion and then continued weekly at a dose of $250 \mathrm{mg} \mathrm{m}^{-2}$ over $60 \mathrm{~min}$. The following pre-medication is administered at each administration: i.v. chlorphenamine $10 \mathrm{mg}$, paracetamol (acetaminophen) $1 \mathrm{~g}$ p.o. and ranitidine $150 \mathrm{mg}$ p.o. Dexamethasone $8 \mathrm{mg}$ i.v. is given on days when oxaliplatin is also given. Patients continue chemotherapy plus cetuximab as in arm A above. Cetuximab may be continued if chemotherapy is stopped because of toxicity or patient choice, but should be discontinued on evidence of disease progression or unacceptable cetuximab toxicity.

Arm C:

Intermittent chemotherapy These patients are treated initially for 12 weeks as in arm A. Patients who have radiologically confirmed progressive disease at this point come off study, as in arms A and B. Patients with stable or responding disease stop chemotherapy and are monitored clinically, at least six weekly, and with CT scans at 12weekly intervals. On evidence of radiological disease progression or on clinical evidence of deterioration, the same chemotherapy is restarted, for a further 12-week course. At that point, treatment is again interrupted. Patients with chemosensitive disease may have an unlimited number of 12-week treatments alternating with treatment breaks. When the patient demonstrates resistance to this treatment as evidenced by progressive disease during a period on chemotherapy, they move on to second-line therapy or supportive care. 


\section{Protocol dose modifications}

Detailed dose reduction and delay protocols are given in the protocol. In general, unresolved grade $\geqslant 2$ toxicity required a 1 -week delay, whereas grade $\geqslant 3$ toxicity or two delays for grade 2 toxicity required a $20 \%$ dose reduction.

\section{Assessments}

Investigator assessments of toxicity are made at six-weekly intervals while on protocol treatment and scored using NCI CTC criteria (version 3.0). Safety is assessed continuously throughout the trial by monitoring of adverse events by the treating physician. Reported serious adverse events are reviewed by an experienced practicing oncologist. All deaths, together with the treating physician's assessment of causality, are also reported. A further assessment of cause of death is also undertaken centrally by an experienced practicing oncologist (TSM, RA or AyM).

\section{Statistical methods}

As arms $\mathrm{A}$ and $\mathrm{C}$ are identical during the first 12 weeks of protocol treatment, they are combined for the purpose of this analysis. All comparisons were tested for significance using Fisher's exact tests. All reported $P$-values are two-sided, and $P$-values less than 0.05 were considered to indicate statistical significance.

\section{Role of the funding source}

The trial is funded by Cancer Research UK, the MRC and supported by an educational grant from Merck Serono. Patient informed consent and data collection were supported by staff from the National Cancer Research Networks across the United Kingdom.

\section{Trial governance}

COIN is an investigator-initiated trial sponsored by the MRC in the United Kingdom. Certain sponsor responsibilities are delegated to the Irish Clinical Oncology Research Group for investigator sites in the Republic of Ireland. Multicentre and Institutional Research Ethics Committee and appropriate regulatory approval have been obtained, and all patients provide written informed consent. Trial management is by the MRC Clinical Trials Unit following the principles of ICH GCP and overseen by an Independent Trial Steering Committee. An Independent Data Monitoring Committee meets at regular intervals (approximately six monthly) for trial oversight, including review of safety data and interim outcome analyses at pre-specified intervals.

\section{RESULTS}

\section{Characteristics of patients}

From March 2005 to July 2006, 804 patients were randomised at 78 centres in the United Kingdom. Baseline characteristics (Table 1) were well balanced across the three arms. Thirty-eight percent received OxMdG and $62 \%$ of patients received Xelox. Patients on average have a high burden of disease at baseline, with approximately $40 \%$ having an unresected or unresectable primary tumour and each patient having on average 2.3 metastatic sites.

\section{Treatment received}

Two hundred and three patients received 5FU plus oxaliplatin (OxMdG, 25\%), 102 received OxMdG + cetuximab (OxMdG + C, $13 \%), 333$ oxaliplatin + capecitabine (Xelox, 41\%) and 166 Xelox + cetuximab (21\%).
Table I Base-line patient and tumour characteristics

\begin{tabular}{|c|c|c|c|}
\hline Treatment arm & $\mathbf{A}$ & B & C \\
\hline Total patients & $269(33 \%)$ & $268(33 \%)$ & 267 (33\%) \\
\hline \multicolumn{4}{|l|}{ Chemotherapy selection } \\
\hline Ox+Cap & 167 (62\%) & $166(62 \%)$ & $166(62 \%)$ \\
\hline OxMdG & 102 (38\%) & 102 (38\%) & $101(38 \%)$ \\
\hline \multicolumn{4}{|l|}{ Sex } \\
\hline Male & $173(64 \%)$ & $167(62 \%)$ & $174(65 \%)$ \\
\hline Female & $96(36 \%)$ & 101 (38\%) & $93(35 \%)$ \\
\hline \multicolumn{4}{|l|}{ Age (years) } \\
\hline$<45$ & $12(4 \%)$ & $18(7 \%)$ & $14(5 \%)$ \\
\hline $45-54$ & $46(17 \%)$ & $34(13 \%)$ & $48(18 \%)$ \\
\hline $55-64$ & $88(33 \%)$ & $100(37 \%)$ & $91(34 \%)$ \\
\hline $65-74$ & $99(37 \%)$ & $95(35 \%)$ & $94(35 \%)$ \\
\hline $75+$ & $24(9 \%)$ & $21(8 \%)$ & $20(7 \%)$ \\
\hline Median age (IQR) & $63(56,69)$ & $63(58,69)$ & $63(55,70)$ \\
\hline \multicolumn{4}{|l|}{ WHO PS } \\
\hline 0 & $120(45 \%)$ & $124(46 \%)$ & $126(47 \%)$ \\
\hline 1 & $128(48 \%)$ & $127(47 \%)$ & $126(47 \%)$ \\
\hline 2 & $21(8 \%)$ & $17(6 \%)$ & $15(6 \%)$ \\
\hline \multicolumn{4}{|l|}{ Current status of primary tumour } \\
\hline Resected & $152(57 \%)$ & | $44(54 \%)$ & $140(52 \%)$ \\
\hline Unresected/unresectable & $103(38 \%)$ & $100(37 \%)$ & $108(40 \%)$ \\
\hline Local recurrence & $14(5 \%)$ & $24(9 \%)$ & $19(7 \%)$ \\
\hline \multicolumn{4}{|l|}{ Metastases } \\
\hline No & $2(1 \%)$ & $2(1 \%)$ & | $(<1 \%)$ \\
\hline Yes & $267(99 \%)$ & $266(99 \%)$ & $266(>99 \%)$ \\
\hline \multicolumn{4}{|l|}{ Type of metastases } \\
\hline Liver only & $55(20 \%)$ & $69(26 \%)$ & $67(25 \%)$ \\
\hline Liver & $197(73 \%)$ & $195(73 \%)$ & $194(73 \%)$ \\
\hline Nodes & $127(47 \%)$ & $109(41 \%)$ & $119(45 \%)$ \\
\hline Lung & $107(40 \%)$ & $103(38 \%)$ & $99(37 \%)$ \\
\hline Peritoneum & $40(15 \%)$ & $40(15 \%)$ & $40(15 \%)$ \\
\hline Other & $38(14 \%)$ & $45(17 \%)$ & $36(13 \%)$ \\
\hline $\begin{array}{l}\text { Mean no. of metastatic sites } \\
\text { at baseline }\end{array}$ & 2.32 & 2.3 & 2.3 \\
\hline \multicolumn{4}{|l|}{ Site of primary tumour } \\
\hline Colon & | 47 (55\%) & 142 (53\%) & $136(51 \%)$ \\
\hline Rectum & $82(30 \%)$ & $88(33 \%)$ & $83(31 \%)$ \\
\hline Rectosigmoid junction & $34(13 \%)$ & $37(14 \%)$ & $48(18 \%)$ \\
\hline Other & $2(1 \%)$ & I $(<1 \%)$ & $0(0 \%)$ \\
\hline Missing data & $4(1 \%)$ & $0(0 \%)$ & $0(0 \%)$ \\
\hline \multicolumn{4}{|c|}{ Prior treatment for patient with metastatic disease } \\
\hline Radiotherapy & $9(3 \%)$ & $6(2 \%)$ & $2(1 \%)$ \\
\hline Surgery & $54(20 \%)$ & $49(18 \%)$ & $53(20 \%)$ \\
\hline
\end{tabular}

PS = performance status.

At least one dose reduction in a COIN trial drug was instituted for toxicity in $28,44,22$ and $49 \%$ of patients in the regimens, respectively. This doubling of dose reductions when chemotherapy was given with cetuximab was highly statistically significant $(P<0.001)$. These broke down as indicated in Table 2 (online). Median dose intensities are as indicated in Table 3 (online). It is of particular note that in patients on Xelox $+\mathrm{C}$, the oxaliplatin dose was reduced in $33 \%$ of patients compared with $15 \%$ on Xelox alone $(P<0.001)$.

\section{Toxicity}

Toxicity data during the first 12 weeks of treatment are available for $97 \%$ of patients. Table 4 (online) indicates all grades of toxicity reported, whereas Table 5 shows grade 3 or 4 toxicities observed 
Table 2 Chemotherapy dose reductions over the first 12 weeks of treatment broken down by regimen and therapeutic agent, on line only

\begin{tabular}{|c|c|c|c|c|c|}
\hline & \multicolumn{2}{|c|}{ Arms $A+C$ (no cetuximab) } & \multicolumn{2}{|c|}{ Arm B (cetuximab) } & Total \\
\hline \multicolumn{6}{|l|}{ Trial drug } \\
\hline $\begin{array}{l}5 \mathrm{FU} \\
\% \text { of patients with } 5 \mathrm{FU} \text { dose reduction }\end{array}$ & $\begin{array}{c}48 \\
(24 \%)\end{array}$ & & $\begin{array}{c}31 \\
(30 \%)\end{array}$ & & $\begin{array}{c}79 \\
\left(26 \%^{\mathrm{a}}\right)\end{array}$ \\
\hline Oxaliplatin & 28 & 50 & 16 & 54 & 148 \\
\hline$\%$ of patients with oxaliplatin dose reduction & $(14 \%)$ & $(15 \%)$ & $(16 \%)$ & $(33 \%)$ & $(18 \%)$ \\
\hline Cetuximab & & & 24 & 33 & 57 \\
\hline$\%$ of patients with cetuximab dose reduction & & & $(24 \%)$ & $(20 \%)$ & $\left(21 \%^{\mathrm{a}}\right)$ \\
\hline Any trial drug & 57 & 73 & 45 & 81 & 256 \\
\hline$\%$ of patients with any trial drug dose reduction & $(28 \%)$ & $(22 \%)$ & $(44 \%)$ & $(49 \%)$ & $(32 \%)$ \\
\hline
\end{tabular}

$5 F U=5$-fluorouracil. On line only. ${ }^{a}$ Denominator is valid subjects only.

Table 3 Dose intensities (first 12 weeks; medians and IQRs)

\begin{tabular}{|c|c|c|c|c|c|}
\hline & \multicolumn{2}{|c|}{ Arms $A+C$ (no cetuximab) } & \multicolumn{2}{|c|}{ Arm B (cetuximab) } & Total \\
\hline \multirow[t]{2}{*}{ Complete data on first round of treatment ( $0-12$ weeks) } & 158 & 247 & 76 & 119 & 600 \\
\hline & Median (IQR) & Median (IQR) & Median (IQR) & Median (IQR) & Median (IQR) \\
\hline Capecitabine & & $96(86,100)$ & & $88(80,100)$ & $93(84,100)$ \\
\hline Oxaliplatin & $89(82,98)$ & $97(89,100)$ & $86(78,94)$ & $92(80,99)$ & $93(83,99)$ \\
\hline Cetuximab, day I & & & $85(78,94)$ & $96(87,100)$ & $92(81,99)$ \\
\hline Cetuximab, day 8 & & & $83(75,92)$ & $93(78,100)$ & $89(77,99)$ \\
\hline Cetuximab, day 15 & & & & $91(75,100)$ & $91(75,100)$ \\
\hline
\end{tabular}

$5 F U=5$-fluorouracil.

Table 4 Dose intensities (first 12 weeks; percentage of patients with $<80 \%$ ) on line only

\begin{tabular}{|c|c|c|c|c|c|}
\hline & \multicolumn{2}{|c|}{ Arms $A+C$ (no cetuximab) } & \multicolumn{2}{|c|}{ Arm B (cetuximab) } & \multirow[b]{2}{*}{ Total } \\
\hline & OxMdG & XELOX & OxMdG & XELOX & \\
\hline Complete data on first round of treatment ( $0-12$ weeks) & $N(\%)$ & $N(\%)$ & $N(\%)$ & $\mathbf{N}(\%)$ & $N(\%)$ \\
\hline Capecitabine & & II (4) & & $17(14)$ & $28(8)$ \\
\hline Oxaliplatin & $5(3)$ & $4(2)$ & $6(8)$ & $14(12)$ & $29(5)$ \\
\hline Cetuximab, day I & & & I (I) & $12(10)$ & $13(7)$ \\
\hline Cetuximab, day 8 & & & $7(9)$ & $23(19)$ & $30(15)$ \\
\hline Cetuximab, day 15 & & & & $29(24)$ & $29(24)$ \\
\hline
\end{tabular}

$5 F U=5$-fluorouracil.

within the first 12 weeks of treatment for the first 804 patients randomised to COIN.

The addition of cetuximab to oxaliplatin- and fluoropyrimidinebased chemotherapy results in an absolute increase in any grade $3 /$ 4 toxicity of approximately $25 \%$ (35-63\% with OxMdG, $35-57 \%$ with Xelox, $P<0.001)$. Grade $3 / 4$ neutropaenia is significantly increased in patients receiving infusional 5FU (17\% without and $26 \%$ with cetuximab) compared with those taking oral capecitabine $(2 \%$ without and $1 \%$ with cetuximab, $P<0.001)$, and this translates into neutropaenic sepsis in $4,5,1$ and $0 \%$, respectively.

Gastrointestinal toxicities, namely nausea, vomiting and diarrhoea, are all significantly increased in patients receiving Xelox 
Table 5 All grades of toxicity reported in the first 12 weeks of treatment

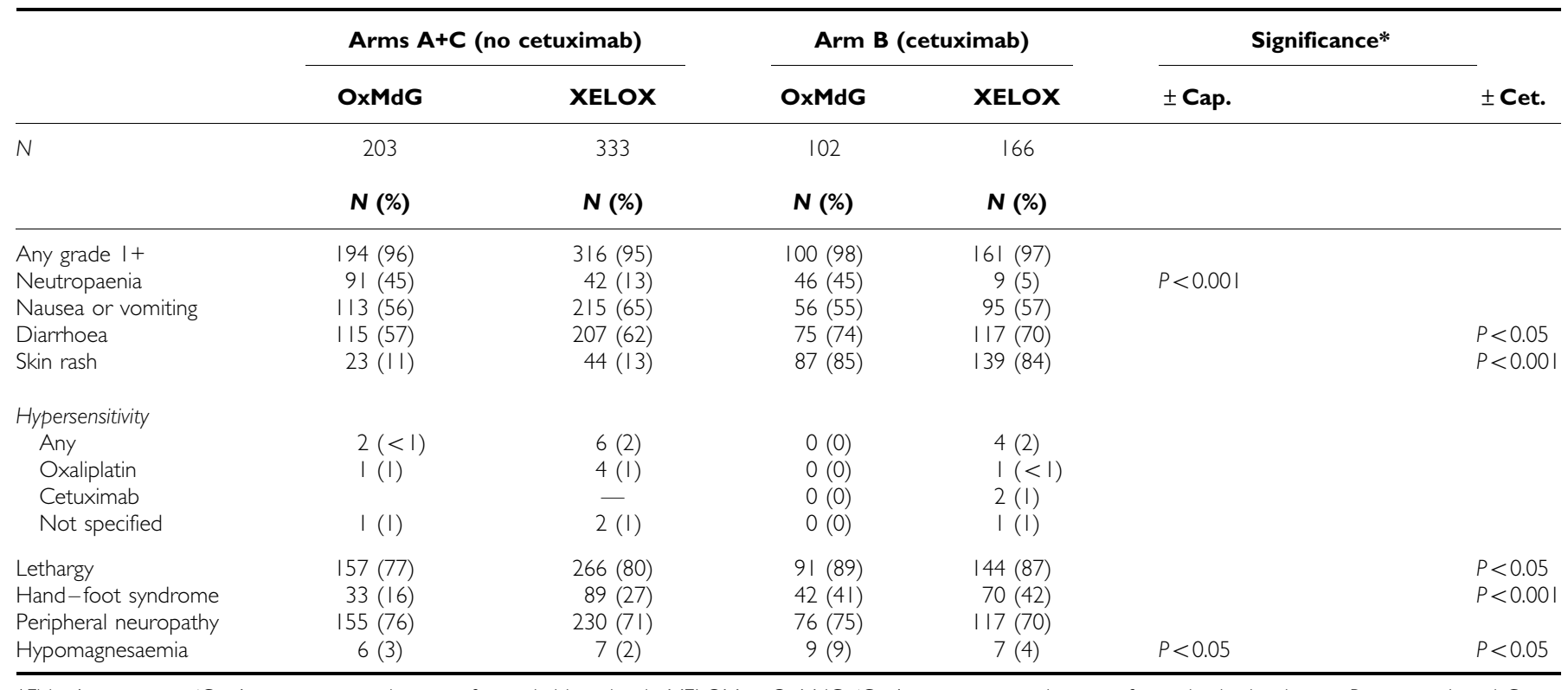

*Fisher's exact test. 'Cap.' = presence vs absence of capecitabine; that is, XELOX vs OXMdG. 'Cet.' = presence vs absence of cetuximab; that is, arm B vs arms A and C.

Table 6

Grade 3 and 4 toxicities reported over first 12 weeks of treatment

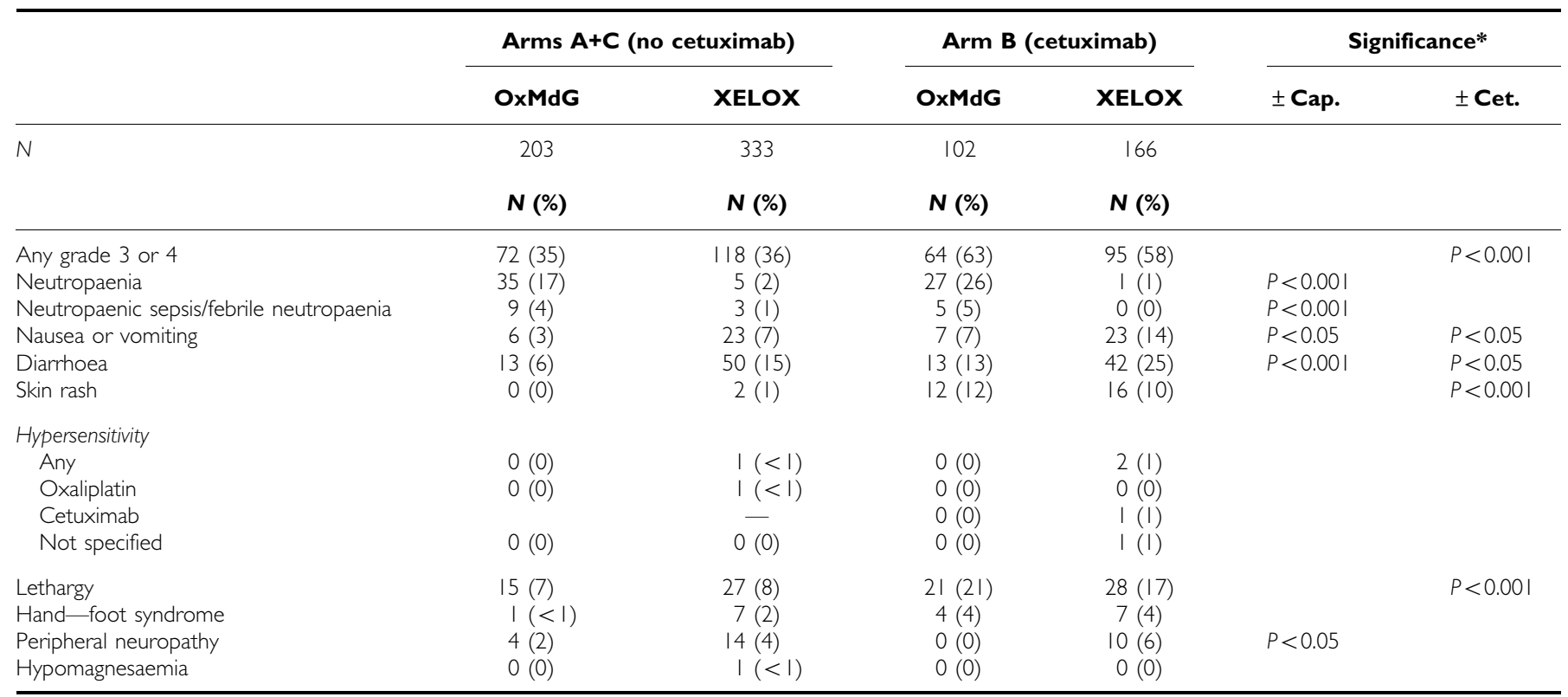

*Fisher's exact test. 'Cap.' = presence vs absence of capecitabine; that is, XELOX vs OxMdG. 'Cet.' = presence vs absence of cetuximab; that is, arm B vs arms A and C.

compared with those receiving OxMdG chemotherapy. These toxicities are further increased by the addition of cetuximab, with an incidence of grade $3 / 4$ diarrhoea of $25 \%$ in patients receiving the Xelox plus cetuximab combination. Statistically significant differences were evident for patients receiving Xelox in combination with cetuximab-based therapy $v s$ Xelox alone for grade 3/4: relative risk (RR) of diarrhoea $1.69(1.17,2.43, P=0.005)$, nausea or vomiting $\mathrm{RR} 2.01(1.16,3.47, P=0.012)$ and lethargy $\mathrm{RR} 2.08$ (1.27, 3.41, $P=0.003)$.

As expected, patients receiving cetuximab have a significantly increased incidence of the skin rash associated with EGFR inhibitors such as cetuximab. This increase is similar irrespective of the chemotherapy regimen $(84 \%$ in patients receiving Xelox + cetuximab and $85 \%$ in patients receiving OxMdG plus cetuximab). Hand - foot syndrome was significantly more common with capecitabine, with $40 \%$ experiencing any grade but only $2-$ $4 \%$ experiencing grade 3 hand-foot syndrome. There was a trend to an increased incidence of hand-foot syndrome in patients treated with cetuximab.

In earlier studies, hypersensitivity reactions have been reported in $1.8 \%$ of patients receiving oxaliplatin chemotherapy, increasing to $4.1 \%$ when cetuximab is added to this chemotherapy regimen (Bokemeyer et al, 2007). In this cohort, there was no such increase in oxaliplatin hypersensitivity with the addition of cetuximab, although numbers are small.

Lethargy is reported in $8 \%$ of patients receiving Xelox and $7 \%$ of patients receiving OxMdG chemotherapy. The addition of cetuximab results in an increase in lethargy, with $17 \%$ of patients 
Table 7 All-cause 60-day mortality

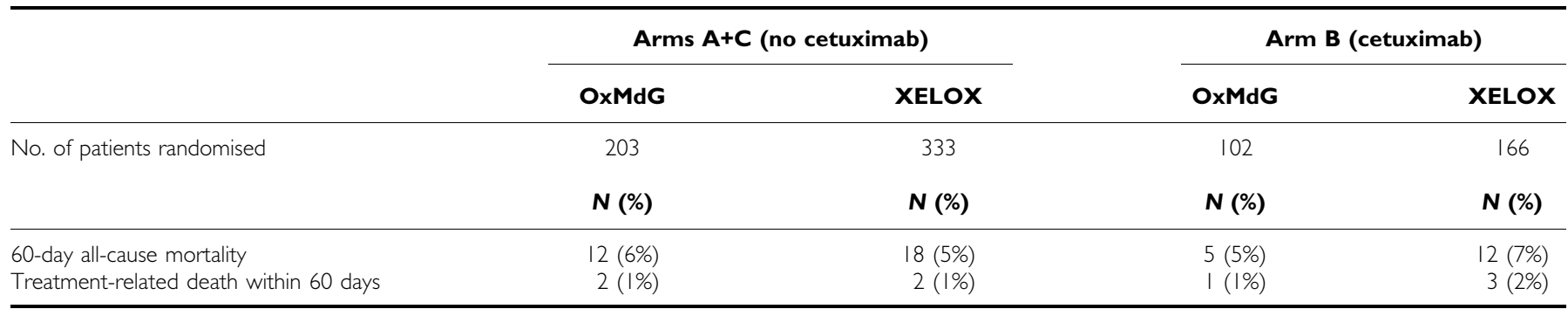

Table 8 A comparison of toxicities among a variety of chemotherapy regimens within four clinical trials in which cetuximab was used

\begin{tabular}{|c|c|c|c|c|c|c|c|c|c|c|}
\hline & XELOX ${ }^{a}$ & $O x M d G^{a}$ & FOLFOX ${ }^{b}$ & $\mathbf{X E L O X}^{\mathbf{b}}$ & FOLFIRI $^{c}$ & FOLFOX-4 & $\begin{array}{c}\text { Cetuximab+ } \\
\text { XELOX }^{\mathrm{a}}\end{array}$ & $\begin{array}{c}\text { Cetuximab+ } \\
\text { OxMdG }\end{array}$ & $\begin{array}{c}\text { Cetuximab+ } \\
\text { FOLFIRI }^{c}\end{array}$ & $\begin{array}{l}\text { Cetuximab+ } \\
\text { FOLFOX }-4^{\text {d }}\end{array}$ \\
\hline$N$ & 333 & 203 & 649 & 655 & 602 & 168 & 166 & 102 & 600 & 170 \\
\hline $\begin{array}{l}\text { Any grade } 3 \text { or } 4 \\
\text { toxicity }\end{array}$ & 35 & 35 & 78.3 & 71.5 & 59.5 & NS & 57 & 63 & 78.0 & NS \\
\hline Neutropaenia & 2 & 17 & 43.8 & 7.0 & 23.3 & 31.5 & 1 & 26 & 26.7 & 27.6 \\
\hline Nausea or vomiting & 7 & 3 & NS & NS & 5.0 & NS & 14 & 7 & 4.5 & NS \\
\hline Diarrhoea & 15 & 6 & 11.2 & 20.2 & 10.5 & 6.0 & 25 & 13 & 15.2 & 7.1 \\
\hline Skin rash & 1 & 0 & NS & NS & 0.2 & 0 & 10 & 12 & 18.7 & 14.1 \\
\hline Hypersensitivity & & & NS & NS & 0 & 1.8 & & & 2.3 & $\begin{array}{l}4.1 \\
2.0\end{array}$ \\
\hline Lethargy & 8 & 7 & NS & NS & 4.5 & 3.0 & 17 & 21 & 5.0 & 3.5 \\
\hline
\end{tabular}

${ }^{\mathrm{a}}$ COIN TRIAL. ${ }^{\mathrm{b}}$ Roche N966. ${ }^{\mathrm{C}}$ Crystal trial. ${ }^{\mathrm{d} O P U S}$ trial.

receiving Xelox plus cetuximab and $21 \%$ of patients receiving OxMdG plus cetuximab experiencing grade $3 / 4$ lethargy within the first 12 weeks of their treatment in the COIN trial.

Peripheral neuropathy is rare in this report, as the time for the report is 'toxicities within the first 12 weeks of therapy' and oxaliplatin neuropathy emerges slightly later than 12 weeks in most patients in whom it occurs. Longer term data will be available when the overall trial outcomes are reported. Similarly in this cohort, hypomagnesaemia is under-reported here because the protocol was modified in October 2005 to make magnesium concentration monitoring and reporting mandatory, by which time most of these patients had commenced treatment.

\section{Deaths within 60 days of randomisation}

Tables 6 and 7 shows the numbers and percentages of deaths within 60 days of randomisation, both all-cause and those attributed to trial treatment. All-cause 60-day mortality is similar irrespective of the chemotherapy regimen or the addition of cetuximab. On review of causation by an experienced practicing oncologist on behalf of the MRC as a sponsor of the trial, treatment-related deaths were not statistically significantly different between treatment regimens.

\section{DISCUSSION}

The COIN trial was designed and completed recruitment during a period when no strong predictor of response to EGFR-inhibiting therapies was known or available. K-ras mutation on tumour samples has since been identified as predictive of minimal likelihood of benefit from the addition of cetuximab in various colorectal clinical settings. However, there is as yet no evidence that somatic $k$-ras status is associated with differences in toxicity of EGFR antibodies or small molecule inhibitors. The initial COIN trial data reported herein encompass the whole eligible population regardless of $k$-ras mutation status. When the outcome data are mature and a full report on (early and late) toxicities accompanies the analysis, data on $k$-ras status for almost all of these patients will be available. It is possible that there may be differences in toxicity that relate to tumour $k$-ras mutational status, but that is not the expectation. The COIN data thus far reflect a typical usage of cetuximab to date in first-line combinations in patients without molecular selection.

A number of interesting toxicity issues are addressed by this study, relating to confirmatory evidence of the differences between oxaliplatin regimens with either capecitabine or $5 \mathrm{FU}$ and to the novel issues of adding cetuximab to either of these regimens.

The findings of the COIN trial are consistent with other international trials including the Roche-sponsored Xelox-1/ NO16966 phase III trial in first-line treatment of colorectal cancer, which reported comparative toxicity for oxaliplatin plus 5FU compared with oxaliplatin plus capecitabine. The COIN trial also shows a significantly higher incidence of neutropaenia (with a $4.8 \%$ incidence of febrile neutropaenia), and half the incidence of diarrhoea with the infusional 5FU regimen compared with capecitabine. Treatment-related mortality in the NO16966 study was $2.1 \%$ with Xelox compared with $1.7 \%$ with FOLFOX (Cassidy et al, 2008) (see Table 8 for comparisons).

The addition of cetuximab to oxaliplatin-based regimens has not been previously reported in a phase III trial, but data from the large OPUS phase II study show no significant uplift in toxicity when combining cetuximab with FOLFOX-4 (a regimen with a lower infused 5FU dose compared with that used in the COIN trial) (Bokemeyer et al, 2007).

The addition of cetuximab to 5FU plus irinotecan (FOLFIRI) was evaluated in the Crystal trial. A minor increase in grade 3 and 4 diarrhoea (from 10 to $15 \%$ ) was seen with the addition of cetuximab (Van Cutsem et al, 2007).

Table 8 shows the comparative toxicities from the COIN, Roche N966, Crystal and OPUS trials. The only studies that have previously reported the addition of capecitabine-containing combinations with cetuximab are the SAKK group, the AIO CRC study group phase II trials and the single-institution study from 
Heraklion (Borner et al, 2006; Heinemann et al, 2007; Souglakos et al, 2007). The SAKK group performed a randomised phase II trial of 74 patients in whom all received Xelox (as used in COIN) on a three-weekly basis and half of the patients received cetuximab weekly; the toxicity data have recently been published and demonstrate a non-significant increase in grade $3 / 4$ diarrhoea from 16 to $22 \%$, with the addition of cetuximab (Borner et al, 2008). The AIO group trial randomised patients to XELIRI or Xelox both with the addition of cetuximab. In the 41 patients receiving Xelox plus capecitabine (in a similar dose and schedule to COIN), grade $3 / 4$ toxicity was seen in $19.5 \%$. The single-centre Heraklion trial recruited 40 patients and used the two-weekly capecitabine plus oxaliplatin (CAPOX) regimen in combination with cetuximab (weekly) with only 7 days of capecitabine. The rate of grade $3 / 4$ diarrhoea in this trial was $7.5 \%$.

The key finding of this report is the synergistic effect on diarrhoeal toxicity of the oxaliplatin, capecitabine and cetuximab combination. This finding had been observed in an earlier IDMC report and the TMG had, on the instruction of the IDMC, improved the patient information cards and fed back the data to investigators so that they include this information in their discussions with patients regarding selection of capecitabine- or 5FU-based treatment. Despite this, the rate of diarrhoea was gradually increasing: for those patients receiving oxaliplatin, capecitabine and cetuximab, a grade $3 / 4$ diarrhoea rate of $23 \%$ was reported in the ASCO abstract (Maughan, 2007) submitted in December 2006 and this had reached 30\% (on the data set from the March IDMC, released to the TMG as of July 2007). Thus, there was no sign of an improvement with greater experience of the regime by investigators despite information to investigators and improved patient information.

This increased toxicity is reflected in the increased incidence of dose reduction in those patients receiving cetuximab. Overall, patients receiving cetuximab had a dose reduction of any agent twice as often as those without cetuximab. In particular, those treated with Xelox + cetuximab had a $33 \%$ incidence of oxaliplatin dose reductions compared with $15 \%$ on Xelox alone. It is possible that such dose reductions may contribute to the impaired outcomes seen in the OPUS trial in the ras mutant sub-group treated with FOLFOX plus cetuximab, and this will be analysed further in the outcomes of the COIN trial.

The trial management group reviewed the data in greater detail and discussed the possible options. The options included first making no protocol change, but urging further diligence in information giving and dose reduction in face of toxicity; dose reduction in the face of grade 2 toxicity rather than grade 3; or alternatively, an immediate dose reduction of capecitabine from 1000 to $850 \mathrm{mg} \mathrm{m}^{-2}$ b.d. from cycle 1 , which was in line with the TREE 2 doses and the Expert $\mathrm{C}$ protocol.

The advantages of this second option were that this would be the safest and swiftest course of action to reduce the toxicity for patients on arm B who choose to use capecitabine as the fluoropyrimidine. In addition, the data from the revised dose would provide evidence for a more manageable combination schedule for the future, which would be consistent with other international data of capecitabine plus oxaliplatin when used in combination with a biological agent. Finally, this would provide for a more equitoxic comparison between arm A (full-dose Xelox) and arm B (dose-reduced Xelox + cetuximab).

The disadvantages were considered to be that we would be using a different regimen for the comparator in arm A (full-dose Xelox) $v s$ arm B (dose-reduced Xelox $+\mathrm{C}$ ). This reduced dose intensity for all subsequent patients on Xelox + cetuximab might impact on the efficacy of this combination. This could in turn cause difficulties in reporting the trial in view of the mid-trial dose reduction for a subset of patients in arm B. The arm B comparisons of toxicity and effectiveness would need to be reported as the total intention-totreat population (1614 patients) and as three subsets: (1) those treated on OxMdG: OxMdG vs OxMdG + cetuximab (c 613 patients on current $38 \%$ usage of OxMdG); (2) those on full-dose Xelox until dose reduction imposed (696 patients); (3) those on modified Xelox comparing full-dose Xelox vs mXELOX + cetuximab (295 patients). On this basis, the TMG considered that the dose reduction at this point in the trial would have a modest effect on the efficacy data, but was required for patient safety.

The option appraisal paper and the toxicity and advised dose reduction data were reviewed by the IDMC and independent TSC and the decision to apply the dose reduction was approved and immediately implemented from July 2007.

\section{CONCLUSION}

This data set is consistent with previous data reporting the toxicity of the addition of cetuximab to combination chemotherapy. There is an increased incidence of grade 3 and 4 toxicities overall. The specific toxicities affect the skin, gastrointestinal tract and lethargy. Infusion-related reactions are relatively rare and can be largely prevented with combination pre-medication regimens. The combination of oxaliplatin $\left(130 \mathrm{mg} \mathrm{m}^{-2}\right.$, i.v., q21d), capecitabine $\left(2000 \mathrm{mg} \mathrm{m}^{-2}\right.$ for 14 out of 21 days) and cetuximab was associated with an unacceptable rate of grade 3,4 diarrhoea, and a dose reduction of capecitabine from 2000 to $1700 \mathrm{mg} \mathrm{m}^{-2}$ day $^{-1}$ has been introduced into the trial protocol and is advised for off protocol use of this triple combination.

\section{ACKNOWLEDGEMENTS}

The COIN trial is supported by a grant from CRUK, UK Medical Research Council funding and an educational grant from Merck Sorono. Professor TS Maughan has received honoraria and research funding from Merck Sorono.

\section{REFERENCES}

Bokemeyer C, Bondarenko I, Makhson A, Hartmann JT, Aparicio J, Zampino M, Donea S, Ludwig H, Zubel A, Koralewski P (2007) Cetuximab plus 5-FU/FA/oxaliplatin (FOLFOX-4) versus FOLFOX-4 in the first-line treatment of metastatic colorectal cancer (mCRC): OPUS, a randomized phase II study. J Clin Oncol 25(S): 4035

Borner M, Koeberle D, Von Moos R, Saletti P, Rauch D, Hess V, Trojan A, Helbling D, Pestalozzi B, Caspar C, Ruhstaller T, Roth A, Kappeler A, Dietrich D, Lanz D, Mingrone W (2008) Adding cetuximab to capecitabine plus oxaliplatin (XELOX) in first-line treatment of metastatic colorectal cancer: a randomized phase II trial of the Swiss Group for Clinical Cancer Research SAKK. Ann Oncol 19: 1288-1292
Borner M, Mingrone W, Koeberle D, Von Moos R, Rauch D, Saletti P, Herrmann R, Dietrich D, Lanz D, Roth A (2006) The impact of cetuximab on the capecitabine plus oxaliplatin (XELOX) combination in first-line treatment of metastatic colorectal cancer (MCC): A randomized phase II trial of the Swiss Group for Clinical Cancer Research (SAKK). J Clin Oncol 24(S): 3551

Cassidy J, Clarke S, Diaz-Rubio E, Scheithauer W, Figer A, Wong R, Koski S, Lichinitser M, Yang TS, Rivera F, Couture F, Sirzen F, Saltz L (2008) Randomised phase III study of capecitabine plus oxaliplatin compared with fluorouracil/folinic acid plus oxaliplatin as first-line therapy for metastatic colorectal cancer. J Clin Oncol 26: 2006-2012 
Cunningham D, Humblet Y, Siena S, Khayat D, Bleiberg H, Santoro A, Bets D, Mueser M, Harstrick A, Verslype c, Chau I, Van Cutsem E (2004) Cetuximab monotherapy and cetuximab plus irinotecan in irinotecanrefractory metastatic colorectal cancer. $N$ Engl J Med 351: 337-345

Heinemann VNM, Vehling-Kaiser U, Stauch M, Oruzio D, Schulze M, Walther J, Weiss J, Dietzfelbinger H, Fischer Von Weikersthal L (2007) XELIRI plus cetuximab versus XELOX plus cetuximab for first-line therapy of metastatic colorectal cancer (CRC): A randomized trial of the AIO CRC Study Group. J Clin Oncol 25(S): 278

http://www-dep.iarc.fr/'Globocan 2002.'(2008)

Maindrault-Goebel F, Lledo G, Chibaudel B Mineur L, Andre T, Bennamoun M, Mabro M, Artru P, Louvet C, de Gramont A (2007) Final results of OPTIMOX2, a large randomized phase II study of maintenance therapy or chemotherapy-free intervals (CFI) after FOLFOX in patients with metastatic colorectal cancer (MRC): A GERCOR study. J Clin Oncol 25(S): 4013

Maughan T, on behalf of the COIN Trial Management Group Investigators Cetuximab (C), oxaliplatin (Ox) and fluoropyrimidine (Fp) (2007) Toxicity during the first 12 weeks of treatment for the first 804 patients entered into the MRC COIN (CR10) trial. J Clin Oncol 25(S): 4070

Maughan TS, James RD, Kerr DJ, Ledermann JA, Seymour MT, Topham C, McArdle C, Cain D, Stephens RJ (2003) Comparison of intermittent and continuous palliative chemotherapy for advanced colorectal cancer: a multicentre randomised trial. Lancet 361: 457-464

Seymour MT, Maughan TS, Ledermann JA, Topham C, James R, Gwyther SJ, Smith DB, Shepherd S, Maraveyas A, Ferry DR, Meade AM, Thompson L, Griffiths GO, Parmar MK, Stephens RJ (2007) Different strategies of sequential and combination chemotherapy for patients with poor prognosis advanced colorectal cancer (MRC FOCUS): a randomised controlled trial. Lancet 370: 143-152

Sobrero AF, Maurel J, Fehrenbacher L, Scheithauer W, Abubakr YA, Lutz MP, Vega-Villegas ME, Eng C, Steinhauer EU, Prausova J, Lenz HJ, Borg
C, Middleton G, Kroning H, Luppi G, Kisker O, Zubel A, Langer C, Kopit J, Burris HA (2008) EPIC: phase III trial of cetuximab plus irinotecan after fluoropyrimidine and oxaliplatin failure in patients with metastatic colorectal cancer. J Clin Oncol 26: 2311-2319

Souglakos J, Kalykaki A, Vamvakas L, Androulakis N, Kalbakis K, Agelaki S, Vardakis N, Tzardi M, Kotsakis AP, Gioulbasanis J, Tsetis D, Sfakiotaki G, Chatzidaki D, Mavroudis D, Georgoulias V (2007) Phase II trial of capecitabine and oxaliplatin (CAPOX) plus cetuximab in patients with metastatic colorectal cancer who progressed after oxaliplatin-based chemotherapy. Ann Oncol 18: 305-310

Therasse P, Arbuck SG, Eisenhauer EA, Wanders J, Kaplan RS, Rubinstein L, Verweij J, Van Glabbeke M, van Oosterom AT, Christian MC, Gwyther SG (2000) New guidelines to evaluate the response to treatment in solid tumours. J Natl Cancer Inst 92: 205-216

Tournigand C, Cervantes A, Figer A, Lledo G, Flesch M, Buyse M, Mineur L, Carola E, Etienne PL, Rivera F, Chirivella I, Perez-Staub N, Louvet C, André T, Tabah-Fisch I, de Gramont A (2006) OPTIMOX1: a randomized study of FOLFOX4 or FOLFOX7 with oxaliplatin in a stop-and-Go fashion in advanced colorectal cancer - a GERCOR study. J Clin Oncol 24: $394-400$

Van Cutsem E, Nowacki M, Lang I, Cascinu S, Shchepotin I, Maurel J, Rougier P, Cunningham D, Nippgen J, Köhne C (2007) Randomized phase III study of irinotecan and 5-FU/FA with or without cetuximab in the first-line treatment of patients with metastatic colorectal cancer (mCRC): The CRYSTAL trial. J Clin Oncol 25(S): 4000

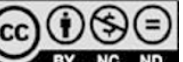

This work is licensed under the Creative Commons Attribution-NonCommercial-NoDerivs 3.0 License. To view a copy of this license, visit http://creativecommons.org/ licenses/by-nc-nd/3.0/. 